\author{
Алексенко Е.Ю., Цвингер С.М. \\ ФГБОУ ВО «Читинская государственная медицинская академия», г. Чита, Россия
}

Резюме. Распространенность артериальной гипертензии у больных остеартрозом достаточно высокая. По данным исследователей, сочетание остеоартроза и артериальной гипертензии регистрируется у 45-80 пациентов в зависимости от возрастных категорий. Патогенетические механизмы формирования и течения артериальной гипертензии у больных остеоартрозом неизменно вызывают интерес у клиницистов. В работе изучены уровни систолического и диастолического артериального давления, содержание некоторых цитокинов (IL-1 $\beta$, IL-6, IL-18, TNF $\alpha$ ), показатели эндотелиальной функции (EDN1, активность vWF) в зависимости от интенсивности боли у 83 больных (средний возраст $45,7 \pm 6,3$ лет) с остеоартрозом коленных и тазобедренных суставов II-III рентгенологической стадии и хроническим болевым синдромом. Выраженность болевого синдрома в течение последнего месяца оценивалась с применением визуальной аналоговой шкалы. По данному показателю пациенты были разделены на три группы. І-ю группу составили 27 пациентов со слабо выраженным болевым синдромом (показатель $\leq 3)$, во ІІІю вошли 34 человека с умеренно выраженной суставной болью $(3<$ показатель < 7), III-я группа состояла из 22 человек, которые оценивали боль как сильную (показатель $\geq 7$ ). Установлено, что увеличение интенсивности болевого синдрома влечет за собой возрастание уровней систолического и диастолического артериального давления. Вместе с тем у больных остеоартрозом при умеренном и выраженном болевом синдроме наблюдалось повышенное содержание провоспалительных цитокинов и EDN1 и возрастание активности vWF. Минимальные значения показателей исследуемых в работе цитокинов отмечены у больных со слабо выраженным болевым синдромом, а максимальные - в группе пациентов с сильной болью и артериальной гипертензией. Медиаторы воспаления обладают способностью вызывать активацию и повреждение эндотелия, ведущие к его дисфункции. Активность vWF и содержание EDN1 увеличивались во всех исследуемых группах при нарастании интенсивности боли. Повышение концентрации EDN1, увеличение активности vWF можно расценить как объективные признаки наличия эндотелиоза у больных остеоартрозом. Эндотелиальная дисфункция, в свою очередь, является одним из ведущих патофизиологических механизмов развития артериальной гипертензии. Это может свидетельствовать о вкладе болевого синдрома

Адрес для переписки:

Алексенко Елена Юрьевна

ФГБОУ ВО «Читинская государственная медицинская академия»

672000, Россия, г. Чита, ул. Горького, 39 .

Тел.: 8 (914) 526-00-98.

Факс: 8(3022) 32-30-58.

E-mail:e-alexe@mail.ru
Address for correspondence:

Aleksenko Elena Yu.

Chita State Medical Academy

672000, Russian Federation, Chita, Gorkogo str., 39 a.

Phone: 7 (914) 526-00-98.

Fax: 7 (3022) 32-30-58.

E-mail:e-alexe@mail.ru

\section{Образец цитирования:}

Е.Ю. Алексенко, С.М. Цвингер «Уровень провоспалительных цитокинов и состояние эндотелиальной функции у больных остеоартрозом в зависимости от интенсивности болџ» // Медицинская иммунология, 2017. Т. 19, № 3. С. 307-312. doi: 10.15789/1563-0625-2017-3-307-312

(C) Алексенко Е.Ю., Цвингер С.М., 2017

\section{For citation:}

E.Yu. Aleksenko, S.M. Tsvinger "Proinflammatory cytokine levels and indexes of endothelial function in patients with osteoarthritis is dependent on the pain intensity”, Medical Immunology (Russia)/Meditsinskaya Immunologiya, 2017, Vol. 19, no. 3, pp. 307-312.

doi: 10.15789/1563-0625-2017-3-307-312

DOI: $10.15789 / 1563-0625-2017-3-307-312$ 
в возникновение и развитие артериальной гипертензии у данной группы больных. Хронический болевой синдром является фактором раннего развития осложнений сердечно-сосудистых заболеваний у пациентов с остеортрозом.

\title{
PROINFLAMMATORY CYTOKINE LEVELS AND INDEXES OF ENDOTHELIAL FUNCTION IN PATIENTS WITH OSTEOARTHRITIS IS DEPENDENT ON THE PAIN INTENSITY
}

\author{
Aleksenko E.Yu., Tsvinger S.M. \\ Chita State Medical Academy, Chita, Russian Federation
}

\begin{abstract}
Prevalence of hypertension in patients with osteoarthritis is rather high. According to published data, a combination of osteoarthritis and hypertension is registered in 45 to $80 \%$ of the patients, dependent on the age group. Pathogenesis of arterial hypertension developing in patients with osteoarthritis is of sufficient interest to the clinicians. We studied systolic and diastolic blood pressure, levels of some cytokines (IL-1 $\beta$, IL-6, IL-18, TNF $\alpha$ ) and markers of endothelial function (EDN1, vWF activity) in 83 patients (mean age $45.7 \pm 6.3$ years) with osteoarthritis of knee and hip joints (radiographic stage II-III) accompanied by chronic pain. Local pain intensity over last month was evaluated by means of a visual analogue scale. According to the data obtained, the patients were divided into three groups. Group 1 consisted of 27 patients with mild pain (the pain index $\leq 3$ points), the $2^{\text {nd }}$ group included 34 persons with moderate pain ( 3 to 7 points), and the $3^{\text {rd }}$ group consisted of 22 people with highest pain ratings (pain index $\geq 7$ ). Increased pain intensity correlated with elevation of systolic and diastolic blood pressure. Meanwhile, higher levels of proinflammatory cytokines, and increased activity of EDN1 and vWF were observed in patients with moderate osteoarthritis and severe pain syndrome. Minimal values of cytokines were observed in patients with mild pain, whereas maximal levels, in the patients with severe pain and arterial hypertension. Inflammatory mediators are able to induce activation and injury of endothelium causing its dysfunction. vWF activity and EDN1 contents increased in all the studied groups, along with increased pain intensity. Higher EDN1 concentration and vWF activity may be considered objective signs of endotheliosis in the osteoarthritis patients. In turn, endothelial dysfunction is among major pathophysiological mechanisms of arterial hypertension. This may suggest a sufficient contribution of pain to occurrence and development of artherial hypertension in this group of patients. Chronic pain is likely to be a factor of early development of cardiovascular complications in the patients with osteoarthritis.
\end{abstract}

Keywords: osteoarthritis, arterial hypertension, cytokines, endothelial function, pain syndrome

\section{Введение}

Распространенность артериальной гипертензии (АГ) у больных остеартрозом (ОА) достаточно высокая. По данным исследователей, сочетание АО и АГ регистрируется у 45-80 пациентов в зависимости от возрастных категорий [7, 9]. Особенности формирования и течения АГ у больных ОА неизменно вызывают интерес у клиницистов. Кроме традиционных факторов риска, специфический вклад в развитие сердечно-сосудистых осложнений у больных ОА могут вносить рецидивирующий болевой синдром, хроническое воспаление, прием нестероидных противовоспалительных препаратов [5, 6]. Доказана позитивная корреляция между отдельными интерлейкинами (IL-1 и IL-6) и уровнем систолического и диастолического атериального давления (АД) [2, 3]. Медиаторы воспаления обладают способностью вызывать активацию и повреждение эндотелия, ведущие к его дисфункции, что убедительно доказано на примере ревматоидного артрита и анкилозирующего спондилита $[8,4]$. Эндотелиальная дисфункция, в свою очередь, является одним из ведущих патофизиологических механизмов развития АГ [10, 11]. Основную роль в развитии дисфункции эндотелия при ОА играют окислительный стресс, продукция мощных вазоконстрикторов (эндотелины, эндопероксиды, АТII), 
а также провоспалительных цитокинов, которые подавляют продукцию оксида азота [1, 2]. Эндотелиальная дисфункция со снижением биодоступности эндотелий-зависимого фактора релаксации - оксида азота и увеличением уровня эндотелина, вероятно, вызывает нарушение микроциркуляции при ОА. Патогенетические связи $\mathrm{AO}$ и АГ в фокусе взаимоотягощения патологии исследованы в настоящее время недостаточно.

Цель работы - изучить содержание некоторых цитокинов, показателей эндотелиальной функции, уровень среднего систолического и диастолического АД у больных ОА в зависимости от выраженности болевого синдрома.

\section{Материалы и методы}

В исследование было включено 83 больных с OA коленных и тазобедренных суставов II-III рентгенологической стадии. Средний возраст

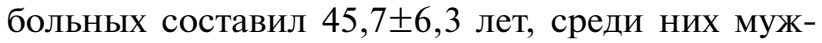
чин было 37, женщин - 46. У всех пациентов наблюдался болевой синдром. Характеристика его была следующая: боли при движении возникали у 83 пациентов (100\%), ночные боли наблюдались у 24 больных (28,9\%), стартовые боли испытывали 28 человек $(33,7 \%)$. Выраженность болевого синдрома в течение последнего месяца оценивалась с применением визуальной аналоговой шкалы (ВАШ, от 0 - отсутствие боли до $10-$ максимально выраженная, нестерпимая боль). По данному показателю пациенты были разделены на три группы. І-ю группу составили 27 пациентов со слабо выраженным болевым синдромом (показатель ВАШ $\leq 3$ ), во II-ю вошли 34 человека с умеренно выраженной суставной болью (индекс был более 3, но менее 7), III-я группа состояла из 22 человек, которые оценивали боль как сильную (по ВАШ более 7).

Всем пациентам проводилось определение усредненных показателей систолического, диастолического артериального давления (САД, ДАД) за сутки при мониторировании АД. Средняя длительность мониторирования АД составила $22,1 \pm 1,3$ часа, при этом интервалы измерения АД днем составляли 15 минут, в ночное время (с $23^{00}$ до $\left.7^{00}\right)-30$ минут. В период проведения СМАД пациенты не были ограничены в физических нагрузках, они продолжали вести обычный образ жизни. За двое суток до и в день проведения исследования пациенты не принимали никаких антигипертензионных препаратов.

Метод определения активности фактора Виллебранда (vWF) основан на его способности вызывать агглютинацию тромбоцитов в присутствии антибиотика ристоцетина (ристомицина).
Способность к такой агглютинации сохраняется у тромбоцитов после их фиксации формальдегидом, когда полностью утрачивается реакция на другие индукторы агрегации. Изучение проводилось с использованием набора реактивов для определения vWF (НПО РЕНАМ, Россия). Количественное определение эндотелина (EDN1) человека в сыворотке проводилось с использованием иммуноферментного набора компании «БиоХимМак», результаты исследования выражались в фмоль/л. Проводилось изучение следующих цитокинов: IL-1 $\beta$, IL-6, TNF $\alpha$ в крови. Для исследования использовались наборы реагентов ЗАО «Вектор-Бест». Результаты исследований определялись в пг/мл.

Статистическая обработка данных проводилась с помощью пакета статистических программ Statistica 6.1. Перед проведением расчетов все вариационные ряды тестировались на нормальность. Если распределение вариационных рядов не подчинялось критериям нормальности, применялись методы непараметрической статистики. Для описания характера распределения количественных признаков определялись средние величины (M), стандартное отклонение (SD). При использовании методов непараметрической статистики определялись медиана, межквартильный интервал (от 25 до 75\%). Достоверность различий оценивалась с использованием t-критерия Стьюдента и с помощью U-критерия МаннаУитни при ненормальном распределении. Различия считались достоверными при $\mathrm{p}<0,05$. Наряду с этим был проведен корреляционный анализ с использованием коэффициента ранговой корреляции Спирмена и статистики тау Кендалла.

\section{Результаты и обсуждение}

При изучении исследуемых параметров у больных с ОА выявлены однонаправленные изменения. Полученные данные представлены в таблице 1. Увеличение интенсивности болевого синдрома влекло за собой возрастание уровней САД и ДАД, при этом достоверная разница получена между I и III группами. Хронический болевой синдром является фактором раннего развития осложнений сердечно-сосудистых заболеваний у пациентов с ОА [6]. При детальном анализе показателей суточного мониторирования АД, анамнеза жизни, данных амбулаторных карт у всех пациентов III-й группы имелась сопутствующая артериальная гипертензия ранее существовавшая или впервые диагностированная. В I группу вошли два больных с сопутствующей артериальной гипертензией 1 степени, во II-й группе таковых было трое. 
ТАБЛИЦА 1. СОДЕРЖАНИЕ НЕКОТОРЫХ ЦИТОКИНОВ, ПОКАЗАТЕЛЕЙ ЭНДОТЕЛИАЛЬНОЙ ФУНКЦИИ, УРОВЕНЬ СРЕДНЕГО САД И ДАД У БОЛЬНЫХ ОА В ЗАВИСИМОСТИ ОТ ВЫРАЖЕННОСТИ БОЛЕВОГО СИНДРОМА

TABLE 1. CONTENTS OF SOME CYTOKINES, ENDOTHELIAL DYSFUNCTION INDEXES, MEAN SYSTOLIC (SAD) AND DIASTOLIC (DAD) ARTERIAL PRESSURE IN OSTEOARTHRITIS PATIENTS DEPENDENT ON SEVERITY OF PAIN SYNDROME

\begin{tabular}{|c|c|c|c|c|}
\hline $\begin{array}{l}\text { Показатель } \\
\text { Index }\end{array}$ & $\begin{array}{l}\text { I группа } \\
\text { Group I } \\
(\mathrm{n}=27)\end{array}$ & $\begin{array}{l}\text { II группа } \\
\text { Group II } \\
(\mathrm{n}=34)\end{array}$ & $\begin{array}{c}\text { III группа } \\
\text { Group III } \\
(\mathrm{n}=22)\end{array}$ & $\begin{array}{c}\text { Уровень } \\
\text { статистической } \\
\text { значимости } \\
\text { Statistical } \\
\text { significance level }\end{array}$ \\
\hline $\begin{array}{l}\text { САД, } \\
\text { мм рт. ст. } \\
\text { Systolic AD, mm Hg }\end{array}$ & $116,2 \pm 8.2$ & $126,3 \pm 7,7$ & $146,4 \pm 12,7$ & $\mathrm{p}_{1-3}<0,00001$ \\
\hline $\begin{array}{l}\text { ДАД, } \\
\text { мм рт. ст. } \\
\text { Diastolic AD, Mm Hg }\end{array}$ & $74,5 \pm 7,2$ & $86,8 \pm 6,8$ & $91,8 \pm 12,7$ & $\mathrm{p}_{1-3}<0,0001$ \\
\hline $\begin{array}{l}\text { EDN1, } \\
\mathrm{fmol} / \mathrm{mL}\end{array}$ & $\begin{array}{c}5,8 \\
{[2,4-11,6]}\end{array}$ & $\begin{array}{c}11,6 \\
{[7,5-12,8]}\end{array}$ & $\begin{array}{c}12,8 \\
{[6-15,8]}\end{array}$ & $\begin{array}{l}\mathrm{p}_{1-2}<0,05 \\
\mathrm{p}_{1-3}<0,05\end{array}$ \\
\hline vWF, \% & $\begin{array}{c}82 \\
{[67-117]}\end{array}$ & $\begin{array}{c}114 \\
{[89-161]}\end{array}$ & $\begin{array}{c}119 \\
{[87-129]}\end{array}$ & $\begin{array}{l}\mathrm{p}_{1-2}<0,05 \\
\mathrm{p}_{1-3}<0,05\end{array}$ \\
\hline $\begin{array}{l}\mathrm{IL}-1 \beta \\
\mathrm{pg} / \mathrm{mL}\end{array}$ & $\begin{array}{c}18,7 \\
{[13,1-20,1]}\end{array}$ & $\begin{array}{c}21,1 \\
{[15,9-25]}\end{array}$ & $\begin{array}{c}25,1 \\
{[21,5-74,2]}\end{array}$ & $\begin{array}{l}\mathrm{p}_{1-2}<0,05 \\
\mathrm{p}_{1-3}<0,05 \\
\mathrm{p}_{2-3}<0,05\end{array}$ \\
\hline $\begin{array}{l}\text { IL-6, } \\
\mathrm{pg} / \mathrm{mL}\end{array}$ & $\begin{array}{c}11,7 \\
{[11,2-14,1]}\end{array}$ & $\begin{array}{c}18,2 \\
{[11,9-21,8]}\end{array}$ & $\begin{array}{c}19,7 \\
{[15-24]}\end{array}$ & $\begin{array}{l}\mathrm{p}_{1-2}<0,05 \\
\mathrm{p}_{1-3}<0,05 \\
\mathrm{p}_{2-3}<0,05\end{array}$ \\
\hline $\begin{array}{l}\text { TNF } \alpha, \\
\mathrm{pg} / \mathrm{mL}\end{array}$ & $\begin{array}{c}9,7 \\
{[8,8-10,8]}\end{array}$ & $\begin{array}{c}11,7 \\
{[7,6-13,8]}\end{array}$ & $\begin{array}{c}13,4 \\
{[10,6-15,6]}\end{array}$ & $\begin{array}{l}\mathrm{p}_{1-2}<0,05 \\
\mathrm{p}_{1-3}<0,05 \\
\mathrm{p}_{2-3}<0,05\end{array}$ \\
\hline
\end{tabular}

По активности vWF и содержанию эндотелина-1 обнаружены различия между I и II, III группами. При этом группы с умеренно выраженным и сильным болевым синдромом не отличались друг от друга. Повышение концентрации EDN1, увеличение активности VWF при усилении болевого синдрома можно расценить как объективные признаки наличия эндотелиоза у больных ОА.

Показатели исследуемых в работе всех цитокинов возрастали от I до III группы. Минимальные значения их отмечены у больных со слабо выраженным болевым синдромом, а максимальные - в группе пациентов с сильной болью и артериальной гипертензией. Статистически значимые различия установлены по уровням IL- $1 \beta$, IL-6, TNF $\alpha$ между всеми группами больных. Повышение концентрации провоспалительных цитокинов (IL-1 $\beta$, IL-6, IL-18, TNF $\alpha$ ) у пациентов с ОА в связи с нарастанием болевого синдрома может свидетельствовать о выраженности воспалительного компонента в патогенезе заболевания. Максимальные значения IL-1 $\beta$, IL-6, TNF $\alpha$ продемонстрировала группа больных ОА с выраженным болевым синдромом в сочетании с АГ. Наличие хронического воспаления, вероятно, является одним из патогенетических факторов возникновения и формирования АГ у данной группы больных. 
Учитывая, что провоспалительные цитокины могут изменять функцию эндотелия сосудов, увеличение их концентрации в крови больных ОА может являться одной из причин развития и прогрессирования АГ у этой группы пациентов. В исследовании установлена связь между наличием у больных ОА и отдельными показателями эндотелиальной дисфункции: активностью vWF (Спирмена $\mathrm{R}=0,609)$, содержанием EDN1 (Спирмена $\mathrm{R}=0,193)$. При прогрессировании ОА и увеличении длительности болезни статистически значимо усиливаются проявления дисфункции эндотелия. Следует отметить, что в третьей группе пациентов не представляется возможным оценить роль каждого из исследуемых факторов, поскольку имеет место коморбидность и взаимоотягощающее влияние хронического воспалительного и болевого синдромов при ОА и артериальной гипертензии.

Таким образом, в исследовании у больных с ОА при умеренном и выраженном болевом синдроме выявлено повышение концентрации провоспалительных цитокинов (IL-1 $\beta$, IL-6, IL-18, TNF $\alpha$ ) и признаки наличия эндотелиоза (нарастание концентрации EDN1, усиление активности vWF). Это может свидетельствовать о вкладе болевого синдрома в возникновение и развитие артериальной гипертензии у данной группы больных. Связь ОА и АГ обусловлена общими патогенетическими механизмами хронического воспаления и боли.

\section{Список литературы / References}

1. Алексенко Е.Ю., Говорин А.В., Цвингер С.М. Нитроксидпродуцирующая функция эндотелия и агрегационная способность тромбоцитов у больных первичным остеоартрозом // Бюллетень Восточно-Сибирского научного центра СО РАМН, 2011. № 1. С. 21. [Aleksenko E.Yu., Govorin A.V., Tsvinger S.M. Nitroxideproductive function of endothelial cells and aggregative ability of thrombocytes in patients with primary osteoarthritis. Byulleten 'Vostochno-Sibirskogo nauchnogo tsentra SO RAMN = Bulletin of the East Siberian Scientific Center SBRAMS, 2011, no. 1, p. 21. (In Russ.)]

2. Алексенко Е.Ю., Говорин А.В. Оценка маркеров воспаления и показатели оксидативного стресса у больных остеоартрозом в сочетании с артериальной гипертензией // Сибирский медицинский журнал (Томск), 2011. T. 26, № 1. С. 54-58. [Alexenko E.Yu., Govorin A.V. Assessment of markers of inflammation and oxidative stress indices in patients with arterial hypertension associated with osteoarthrosis. Sibirskiy meditsinskiy zhurnal (Tomsk) = The Siberian Medical Journal (Tomsk), 2011, Vol. 26, no. 1, pp. 54-58. (In Russ.)]

3. Анкудинов А.С., Калягин А.Н. Иммуномодулирующие цитокины при хронической сердечной недостаточности, ассоциированной с остеоартрозом коленных суставов // Сибирский медицинский журнал (Иркутск), 2015. № 5. С. 43-46. [Ankudinov A.S., Kalyagin A.N. Immunomodulatory cytokines in chronic heart failure associated with knee osteoarthritis. Sibirskiy meditsinskiy zhurnal (Irkutsk) = The Siberian Medical Journal (Irkutsk), 2015, no. 5, pp. 43-46. (In Russ.)]

4. Линева О.Г., Попкова Т.В., Новикова Д.С., Насонов Е.Л. Ингибиторы ФНО- $\alpha$ и их влияние на факторы риска поражения сердечно-сосудистой системы у пациентов с ревматоидным артритом // Научнопрактическая ревматология, 2010. T. 48, № 1. С. 31-36. [Lineva O.G., Popkova T.V., Novikova D.S., Nasonov E.L. Tumor necrosis factor- $\alpha$ inhibitors and their effects on cardiovascular risk factorsin patients with rheumatoid arthritis. Nauchno-prakticheskaya revmatologiya $=$ Rheumatology Science and Practice, 2010, Vol. 48, no. 1, pp. 3136. (In Russ.)]

5. Мартынов А.И., Наумов А.В., Верткин А.Л. Ведение больных остеоартритом с коморбидностью в общей врачебной практике: мнение экспертов, основанное на доказательной медицине // Лечащий врач, 2015. № 4. C. 344-349. [Martinov A.I., Naumov A.B., Vertkin A.L. Observation of the patients with osteoarthritis with comorbidity in general medical practice: expert opinions based on evidentiary medicine. Lechashchiy vrach = Attending Doctor, 2015, no. 4, pp. 344-349. (In Russ.)]

6. Мендель О.И., Наумов А.В., Верткин А.Л. Остеоартроз как фактор риска кардиоваскулярных катастроф // Украинский ревматологический журнал, 2010. Т. 3, № 41. С. 13-16. [Mendel O.I., Naumov A.B., Vertkin A.L. Osteoarthritis as a factor of risk of cardiovascular accidents. Ukrainskiy revmatologicheskiy zhurnal = Ukrainian Rheumatology Journal, 2015, Vol. 3, no. 4, pp. 13-16. (In Russ.)]

7. Насонов Е.Л., Попкова Т.В. Кардиоваскулярные проблемы в ревматологии // Научно-практическая ревматология, 2004. № 4. С. 4-9. [Nasonov E.L., Popkova T.V. Cardiovascular problems of rheumatology. Nauchnoprakticheskaya revmatologiya $=$ Rheumatology Science and Practice, 2004, no. 4, pp. 4-9. (In Russ.)]

8. Попкова Т.В., Новикова Д.С., Насонов Е.Л. Сердечно-сосудистые заболевания при ревматоидном артрите // Научно-практическая ревматология, 2016. T. 54, № 2. С. 46-49. [Popkova T.V., Novikova D.S., 
Nasonov E.L. Cardiovascular diseases in rheumatoid arthritis: latest data. Nauchno-prakticheskaya revmatologiya $=$ Rheumatology Science and Practice, 2016, Vol. 54, no. 2, pp. 46-49. (In Russ.)]

9. Griffin T.M., Guilac F. The role of mechanical loading in the onset and progression of osteoarthritis. Exerc. Sport. Sci. Rev., 2005, Vol. 33, no. 4, pp. 195-200.

10. Nüesch E., Dieppe P., Reichenbach S. All cause and disease specific mortality in patients with knee or hip osteoarthritis: population based cohort study. BMJ, 2011, Vol. 342, pp. 11-65.

11. Ong K.L., Wu B.J., Cheung B.M. Arthritis: its prevalence, risk factors, and association with cardiovascular diseases in the United States, 1999 to 2008. Ann. Epidemiol., 2013, Vol. 23, pp. 80-86.

\section{Авторы:}

Алексенко Е.Ю. - д.м.н., доцент, заведующая кафедрой поликлинической терапии с курсом медицинской реабилитации, ФГБОУ ВО «Читинская государственная медицинская академия», г. Чита, Россия

Цвингер С.М. - к.м.н., ассистент кафедры поликлинической терапии с курсом медицинской реабилитации, ФГБОУ ВО «Читинская государственная медицинская академия», г. Чита, Россия

\section{Authors:}

Aleksenko E.Yu., PhD, MD (Medicine), Head, Department of Policlinical Therapy with a Course of Medical Rehabilitation, Chita State Medical Academy, Chita, Russian Federation

Tsvinger S.M., PhD (Medicine), Assistant Professor, Department of Policlinical Therapy with a Course of Medical Rehabilitation, Chita State Medical Academy, Chita, Russian Federation
Поступила 19.10.2016

Отправлена на доработку 20.10.2016

Принята к печати 26.12.2016
Received 19.10.2016

Revision received 20.10.2016

Accepted 26.12.2016 